\title{
BMJ Open Can paramedics use FRAX (the WHO Fracture Risk Assessment Tool) to help GPs improve future fracture risk in patients who fall? Protocol for a randomised controlled feasibility study
}

\author{
Shane Clarke, ${ }^{1}$ Rachel Bradley, ${ }^{1}$ Bethany Simmonds, ${ }^{1}$ Chris Salisbury, ${ }^{2}$ \\ Jonathan Benger, ${ }^{3}$ Elsa Marques, ${ }^{4}$ Rosemary Greenwood, ${ }^{1}$ Lee Shepstone, ${ }^{5}$ \\ Maria Robinson, ${ }^{6}$ John Appleby-Fleming, ${ }^{6}$ Rachael Gooberman-Hill ${ }^{7}$
}

To cite: Clarke S, Bradley R, Simmonds B, et al. Can paramedics use FRAX (the WHO Fracture Risk Assessment Tool) to help GPs improve future fracture risk in patients who fall? Protocol for a randomised controlled feasibility study. BMJ Open 2014;4:e005744 doi:10.1136/bmjopen-2014005744

- Prepublication history and additional material is available. To view please visit the journal (http://dx.doi.org/ 10.1136/bmjopen-2014005744).

Received 27 May 2014 Revised 4 August 2014 Accepted 7 August 2014

\section{CrossMark}

For numbered affiliations see end of article.

Correspondence to

Dr Shane Clarke;

s.clarke@bristol.ac.uk

\section{ABSTRACT}

Introduction: Currently identification, and therefore, management of patients at risk of osteoporotic fracture in the UK is suboptimal. As the majority of patients who fracture have fallen, it follows that people who fall can usefully be targeted in any programme that aims to reduce osteoporotic fracture. Targeting vulnerable patients who are likely to benefit from intervention may help shift the management of fracture prevention into primary care, away from emergency departments. Paramedics who attend to patients who have fallen may be well placed to assess future fracture risk, using the Fracture Risk Assessment Tool (FRAX) and communicate that information directly to general practitioners (GPS).

Methods and analysis: This feasibility study takes the form of a pragmatic, randomised controlled trial aimed at exploring and refining issues of study design, recruitment, retention, sample size and acceptability preceding a large-scale study with fracture as the end point. Patients (aged $>50$ ) who fall, call an ambulance, are attended by a study paramedic and give verbal consent will be asked FRAX and fall questions. Patients who subsequently formally consent to participation will be randomised to control (usual care) or intervention groups. Intervention will constitute transmission of calculated future fracture risk to the patients' GP with suitable, evidence-based recommendations for investigation or treatment. 3 months after the index fall, data (proportion of patients in each group undergoing investigation or starting new treatment, quality of life and health economic) will be collected and analysed using descriptive statistics. A nested qualitative study will explore issues of acceptability and study design with patients, paramedics and GPs.

Ethics and dissemination: This protocol was approved by NRES Committee South Central Oxford C in October 2012. Research Ethics Committee ref.12/SC/ 0604. The study findings will be disseminated through peer-reviewed journals, conference presentations and local public events. A publication plan and authorship criteria have been preagreed.

Trial registration number: ISRCTN: 36245726.

\section{INTRODUCTION}

Osteoporotic fractures are increasingly frequent, with an incidence over 300000 annually in the UK. ${ }^{1}$ Such fractures are often painful and disabling. ${ }^{2}$ Hip fractures are associated with greatest disability and may be predicted by prior fragility fractures at the wrist or spine. $^{2}$ Despite this, only a third of patients who experience such breaks are prescribed treatment to reduce future fracture risk. ${ }^{1}$ The identification, and therefore, management of patients at risk of osteoporotic fracture in the UK at present is suboptimal. ${ }^{1}$

Falls occur increasingly frequently with age. In total, $35 \%$ of people aged over 65 fall each year rising to $50 \%$ at age $85 .^{3}$ Many patients fall repeatedly. $56 \%$ of patients attending an emergency department reported recurrent falls, and $55 \%$ of patients presenting with an acute fracture had previously fallen. ${ }^{5}$ A fall with osteoporosis is particularly risky. In a study of 2649 postmenopausal women, those with osteoporosis were 2.8 times more likely to fracture than those without, and osteoporotic women with a history of fall 24.8 times more likely, even after taking age and body mass index into account. ${ }^{6}$ Treatment to reduce fracture risk in those with osteoporosis is known to be cost-effective $^{78}$ but there is far less evidence to suggest benefit in targeting people who fall for fracture risk assessment. ${ }^{9}$ A high proportion of people who fall and call an ambulance are not reviewed by their general practitioner (GP). Only $25 \%$ of such patients visit their general practice within 3 months of a fall. ${ }^{10}$

Treatment of patients considered at high risk of fracture might consist of a 
combination of non-medication (education, physiotherapy, balance classes) and medication options. Fracture rates may be reduced by calcium and vitamin D supplementation among older people who live in care homes. ${ }^{11}$ Specific treatments such as the bisphosphonates (for example, oral alendronate or intravenous zoledronate) can reduce future fracture rate between $40 \%$ and $50 \%$. $^{7}$

Drug treatment thresholds, based on a combination of fracture risk, age and gender, have been set in the UK by the National Osteoporosis Guideline Group (NOGG). ${ }^{12}$ Both National Institute for Health and Care Excellence (NICE) and NOGG recognise that falls play an important part in fracture risk, but neither include fall history in their risk-profiling algorithms.

However, NICE has published guidance to help clinicians identify patients most at risk of fragility (low impact or osteoporotic) fracture. ${ }^{13-16}$ Technology Appraisal Guidelines (TAG) $160 / 161^{13} 14$ advise the use of clinical factors (family history of hip fracture, conditions associated with osteoporosis, early menopause, excessive alcohol consumption) to target dual energy X-ray absorptiometry (DXA) scans and/or treatment to postmenopausal women. Healthcare commissioners are charged with ensuring NICE guidance is implemented nationally. However the advice is complex (particularly TAG160) and uptake has been poor. ${ }^{1}$ NICE guidance also specifically excludes women taking corticosteroids, and men.

Assessment of future fracture risk is central to good management, and the Fracture Risk Assessment Tool (FRAX) has been developed to enable identification of individuals most at risk of fragility fracture. ${ }^{17}$ FRAX is a validated and freely available algorithm in the form of a calculator that estimates a 10-year absolute risk of fragility fracture from patient data inputted online. The online calculator is clear, easy to use and allows risk calculation in postmenopausal women, patients taking corticosteroids and men aged 50 years or over.

As the majority of patients who fracture have fallen, it follows that people who fall can usefully be targeted in any programme to reduce osteoporotic fracture. Targeting such a population may allow the identification of vulnerable patients who are likely to benefit from intervention, and serve to further shift the burden of fracture prevention into primary care. As people who fall are often attended by paramedics, ${ }^{10}$ involving these professionals in the assessment of future fracture risk has the potential to impact on follow-up and management. Specifically, if paramedics help to determine a patient's 10-year fracture risk at the time of a fall, if that information is conveyed to their GP, and if the GP acts on the recommendations, then a higher proportion of patients may be treated according to the national fracture prevention guidelines.

It is possible that introducing routine FRAX assessment by paramedics of all patients who fall and call an ambulance could reduce long-term fracture rates. In order to justify such a service development, evidence of fracture reduction is required. A large (expensive) trial, conducted over a number of years would be needed to address this important question. Before such an undertaking, it is wise to assess the feasibility of such an approach, to explore the possibilities and potential pitfalls of the intervention, to inform the design of a large-scale randomised trial aimed at reducing fragility fracture rates over the coming years in those who fall.

\section{AIIM}

Our future aim is to determine whether FRAX administered opportunistically by paramedics to people who fall improves diagnosis and treatment of patients at high risk of fracture, leading to a reduced fracture rate. To design such a trial (in the future), we need to conduct a feasibility study to collect data (quantitative and qualitative) to inform the effective design of the main (fracture reduction) trial.

\section{OBJECTIVES}

To achieve the aim of the feasibility study we have set the following objectives:

1. To find out if paramedics are in a position to ask the necessary questions and gather the right information to be able to reliably calculate fracture risk.

2. To determine if the paramedics will collect the information (and submit it) in a busy service setting, and if not, what barriers might exist.

3. To determine recruitment, eligibility and consent rates, and the proportion of participants who continue to study completion.

4. To establish the proportion of patients who are eligible for treatment to reduce fracture risk according to the national fracture prevention guidance.

5. To collect demographic (gender, age) data and information on the relative rate of GP attendance, referral for investigation (DXA scan) or treatment (with reference to the NICE/NOGG guidance), between the intervention and control group.

6. Identify, measure and value resources required to deliver the intervention.

7. Develop the methods to collect resources used in the National Health Service (NHS) for the follow-up period, including retrieving resource-use data from GP practices and hospitals.

8. Collect the patient-completed resource use questionnaires at follow-up to allow an economic evaluation from the perspective of the healthcare user in a future trial.

9. Collect the number of patients completing the generic quality-of-life Euroqol $^{18}$ (EQ-5D-5L) questionnaire at baseline and follow-up.

10. To determine the acceptability of the trial design and intervention for the patients (and carers where appropriate), paramedics and GPs. 
Our objectives will contribute to our aim (above) to design a well-informed, adequately sized, robust, multicentre randomised controlled trial (RCT), to determine whether the intervention has the capacity to increase the proportion of patients at high risk of fracture prescribed treatment in keeping with existing national guidelines.

\section{METHODS AND ANALYSIS \\ Study design}

The feasibility study will take the form of a pragmatic, RCT (allocation ratio 1:1) to explore and refine issues of study design, recruitment, retention, sample size and acceptability to inform a future, multicentre, appropriately powered RCT. It will include assessment using quantitative and qualitative approaches.

The proposed intervention is a service development in which a patient's GP is informed about their fall and their future fracture risk, as assessed at the time of their fall using FRAX.

In practice, a specialist osteoporosis or falls nurse (or equivalent) will use the data collected by paramedics to formulate advice for the GP, however, for the purposes of this feasibility study, the place of the nurse will be taken by a member of the research staff (figure 1).

\section{Setting}

The study will be carried out by paramedics based at an urban ambulance station (study site may be obtained by contacting the corresponding author).

\section{Target population}

Men and women aged 50 or over who have fallen and called an ambulance.

\section{Inclusion and exclusion criteria}

We will include:

1. Men and women aged over 50 who fall (inside or outside their place of residence), call an ambulance and are attended by study paramedics. Men and women under the age of 50 who fall are unlikely to experience a fragility fracture.

We will exclude:

2 Patients who, in the paramedic's opinion, are medically unstable and for whom it would not be appropriate to delay treatment to ask study questions (for example, where the paramedics suspect a cardiac incident).

3 Patients who have fallen in a public place and, in the opinion of the treating paramedics should be conveyed without delay.

4 Patients who are deemed to lack capacity (according to the Mental Health Act (2005)) but for whom there is no available carer or consultee.

5 Patients whose home address is outside the defined urban and suburban catchment.
6 Patients who are admitted to hospital for $24 \mathrm{~h}$ or more. These patients will need to be excluded by the researcher prior to consent (pre-existing service arrangements cover inpatients and our principal target population is in primary care).

We will continue to monitor patients who fall twice or more during the study period, but count the first fall as the index event. Only the first eligible fall will trigger entry into the study.

\section{Intervention}

The intervention will be the calculation of future fracture risk of patients who fall, and the transmission of clear, tailored advice (based on national guidance) regarding investigation and treatment, to the patient's GP.

\section{Control}

Usual care. This means participants are free to visit their GP, or seek further help after their fall, but that we will not prompt such an action. We will not calculate fracture risk in these patients.

All patients (intervention and control) will receive a leaflet about falls phrased in general terms and approved by patient research partners.

\section{Recruitment and sample size}

Paramedics working at the nominated ambulance station attend about 4200 people who fall, each year. The Trust employs 100 full time equivalent (FTE) staff. Each FTE will attend an average 0.8 falls each working week, or between 1 and 2 fallers per ambulance/week.

If $25 \%$ of patients are eligible and consent to inclusion, it will take 20 paramedics 25 working weeks to recruit 100 participants. Paramedics will seek to collect information from around 400 eligible patients (figure 2).

\section{Potential for discrimination between control and intervention groups}

There is no prospective data linking DXA-determined osteoporosis with people who fall. Evidence from heel scans suggests $42 \%$ women ( $>65$ years) who fall have osteoporosis. ${ }^{19}$ We might therefore expect about 15-20 patients in the intervention group to be eligible for treatment.

\section{Paramedic professional development}

We will provide professional development sessions to approximately 20 volunteer paramedics, employed by SWAFT to take part in this research. The paramedics will receive overtime payments to attend. The sessions will be carried out in small groups (4-6), face to face, and facilitated by an experienced clinician according to a preagreed session plan. The paramedics will learn about research, receive refresher training in the Mental Capacity Act, find out about the importance of osteoporosis and learn how to use the FRAX tool. There will be an interactive session designed to allow the paramedics to try out the study 


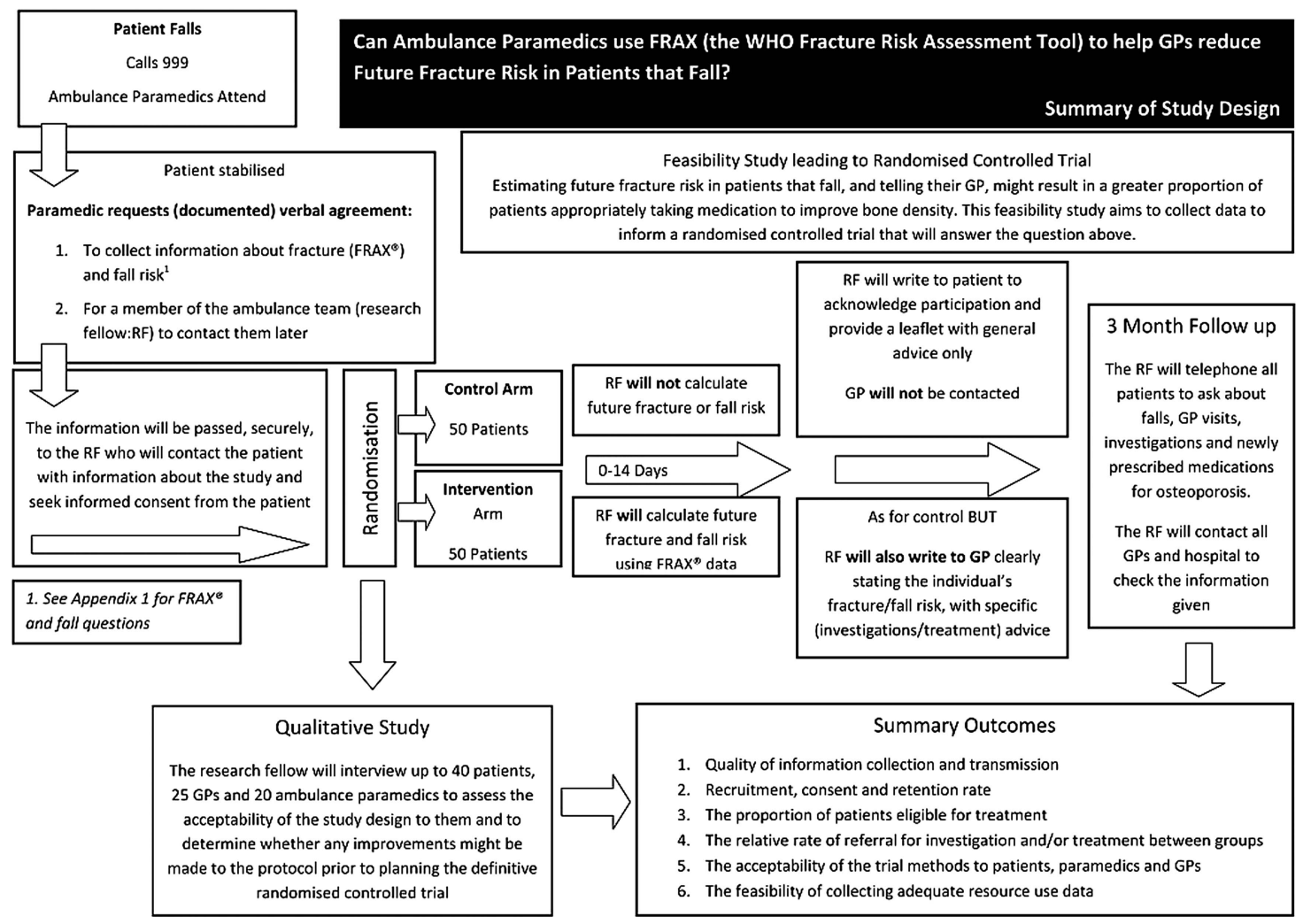

Figure 1 A summary of study design (FRAX, Fracture Risk Assessment Tool; GP, general practitioner).

paperwork, in particular, asking the FRAX questions of a volunteer (drawn from the patient participation group). After the time to ask questions, the paramedic will be appraised in their use of the study literature.

\section{Study plan}

Paramedics will ensure the patient is medically stable and comfortable. If the patient is eligible (see inclusion and exclusions 1-4) the paramedic will seek verbal agreement from the patient to ask FRAX questions relating to osteoporosis risk and falling, and for a member of the research team to contact them later about the study. Paramedics will defer to the patient's carer or consultee for verbal agreement if the patient lacks capacity.

The paramedic will use a double-sided sheet to record the patient's verbal consent on one side, and the answers to questions about fracture (FRAX) and fall risk on the other (see online supplementary appendix 1 ). He or she will then return the completed paper sheets with the (medication) response bag, at the end of shift, to the ambulance base.

The patient contact sheets will be transmitted securely to the researcher. The researcher will then check that potential participants have not been admitted to hospital for $24 \mathrm{~h}$ or longer (exclusion 5).

Within 10 working days of the index fall the researcher will send an information pack (patient information (see online supplementary appendix 2), reply slip, consent form, stamped addressed return envelope) to the patient. The researcher will also then telephone the patient to discuss the study. This will provide an opportunity for the patient to ask any questions they may have in order for them to provide valid, written consent. If the patient prefers to consent in person the researcher will visit the patient.

If the patient declines, we will call the patient to enquire after the reason(s) they have opted not to take part. Thereafter, no further contact will be made. A postal reminder will be sent if we are unable to make contact by telephone and the patient does not return the reply slip. Following written consent, patients will be randomly allocated remotely by Bristol Randomised Trials Collaboration (http://bristol.ac.uk/cobm/research/ brtc.html) to the intervention or control group, by telephone. Enrolment and allocation will be carried out by the research fellow. Patients in both arms will receive acknowledgment of their participation in the trial, and a leaflet containing general information about falls. A EQ-5D-5L ${ }^{18}$ questionnaire will also be included. This is a general measure of a health-related quality-of-life tool that yields a health status quality-of-life score.

With the participants' consent, we will write to their GPs. GPs will be informed of their patient's participation in the study, Primary Care Research Network (PCRN) endorsement of the project, and that funding is available to cover the additional practice time required. We will not write to participants' GPs who have been allocated to the control arm until 3 months after the index fall. 


\section{Can Ambulance Paramedics use FRAX ${ }^{\circledR}$ (WHO Fracture Risk Assessment Tool) to help GPs reduce Future Fracture Risk in Patients who Fall?}

\section{Recruitment, Consent, Randomisation and Follow up}

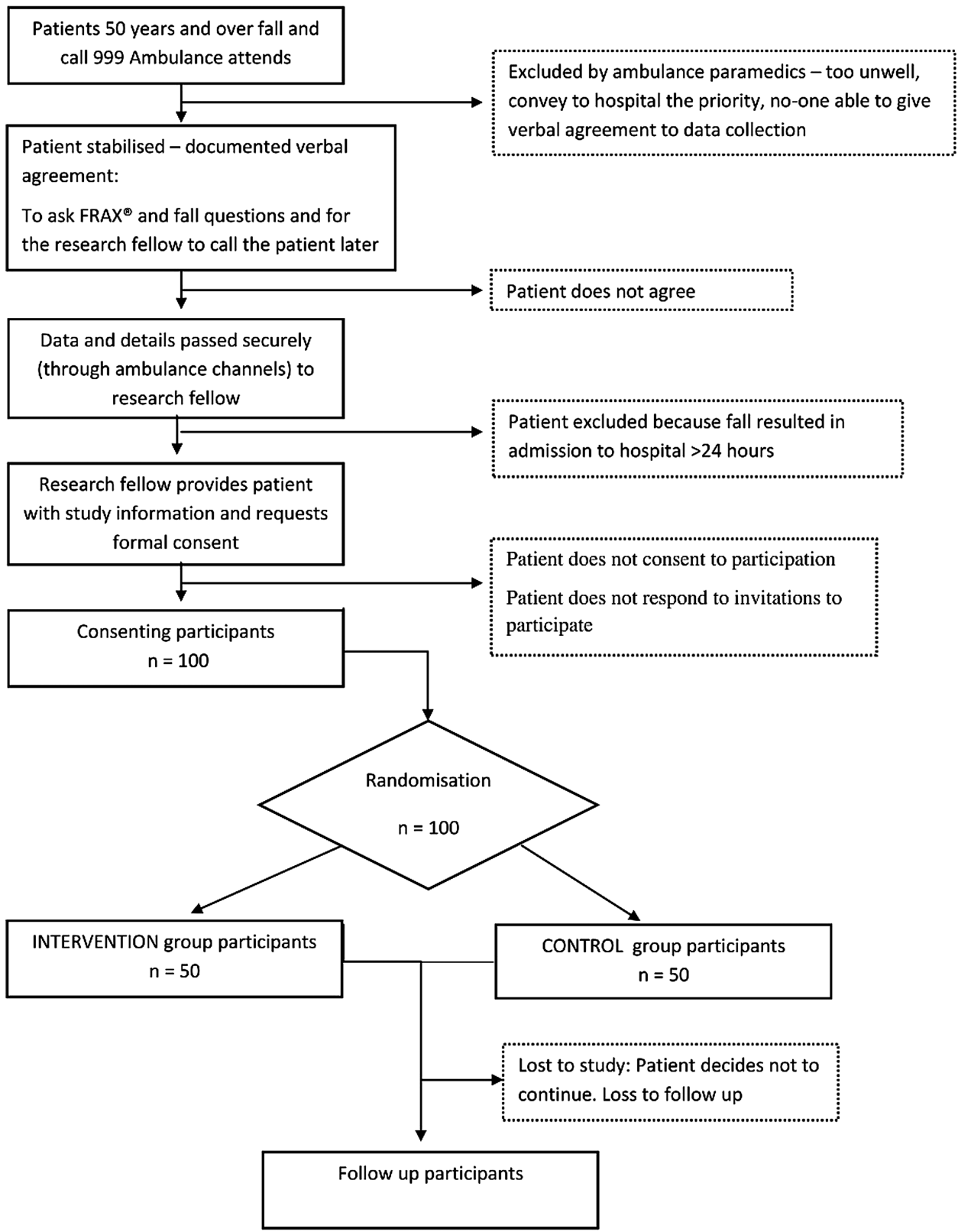

Figure 2 Flow chart showing consent, randomisation and follow-up process (FRAX, Fracture Risk Assessment Tool; GP, general practitioner).

Patients in the intervention arm will have their future fracture risk calculated using FRAX. ${ }^{17}$ A clinical member of the research team will write to the patient's GP with clear advice based on NICE Technology Appraisal Guideline 160 and 161 (Primary and Secondary Prevention of Osteoporotic Fracture $)^{13} 14$ for postmenopausal women (not taking corticosteroids), and from NOGG for postmenopausal women and men. ${ }^{12}$

Where investigation or treatment for osteoporosis is recommended we will include supporting documentation in the pack (for example, a request form for DXA, patient information about osteoporosis, treatments and contact numbers for the local National Osteoporosis Group and advice line).

We will initiate follow-up 3 months after the index fall. We will telephone patients in the intervention and control arms to ask questions about their health, investigations and new treatments. We will also contact the patients' GPs with similar enquiries. Patients will be asked to complete the quality-of-life questionnaire (EQ-5D-5L) for a second time. 
The researcher will check emergency department data and hospital-based medical records to confirm reported hospital attendances, investigations, existing and newly prescribed or recommended treatments.

Table 1 provides a summary of data collection at study entry and 3-month follow-up.

We will send a study report to all patients who participated but we do not intend to send patients allocated to the control group specific information about their fracture risk.

\section{Outcome measures}

The outcome measures for this feasibility study will be:

1. The percentage of FRAX and fall-related data correctly collected by paramedics and the percentage of that information translated to clear advice and received by the correct GP.

2. The eligibility, recruitment and consent rates, and the proportion of participants who continue to study completion.

3. The proportion of patients (men and women) who fall and who are eligible for, but not taking, treatment to reduce fracture risk according to the national fracture prevention guidance.

\section{Table 1 Summary of data collection}

\begin{tabular}{|c|c|c|}
\hline Concept & Measure & Time point(s) \\
\hline Sociodemographics & & $\begin{array}{l}\text { Baseline and } \\
3 \text { months }\end{array}$ \\
\hline Fracture and fall risk & $\begin{array}{l}\text { FRAX and falls } \\
\text { questionnaire }\end{array}$ & $\begin{array}{l}\text { Baseline and } \\
3 \text { months }\end{array}$ \\
\hline $\begin{array}{l}\text { Health-related quality of } \\
\text { life (optional) }\end{array}$ & EQ-5D-5L & $\begin{array}{l}\text { Baseline and } \\
3 \text { months }\end{array}$ \\
\hline $\begin{array}{l}\text { Resource use } \\
\text { questionnaire (optional) }\end{array}$ & Pro forma & 3 months \\
\hline $\begin{array}{l}\text { Fall and fracture } \\
\text { treatments }\end{array}$ & Pro forma & 3 months \\
\hline $\begin{array}{l}\text { Hospital visits and } \\
\text { treatments }\end{array}$ & Pro forma & 3 months \\
\hline $\begin{array}{l}\text { Resources required to } \\
\text { train the paramedics }\end{array}$ & Pro forma & $\begin{array}{l}\text { At training } \\
\text { sessions }\end{array}$ \\
\hline $\begin{array}{l}\text { Resources required } \\
\text { administer the FRAX } \\
\text { tool by paramedics to } \\
\text { fallers }\end{array}$ & Pro forma & $\begin{array}{l}\text { At ambulance } \\
\text { call home visit } \\
\text { to faller }\end{array}$ \\
\hline $\begin{array}{l}\text { Resources required to } \\
\text { compute the FRAX } \\
\text { score to intervention } \\
\text { patients and send } \\
\text { letters to GPs }\end{array}$ & Pro forma & $\begin{array}{l}\text { Post } \\
\text { ambulance } \\
\text { visit, in back } \\
\text { office }\end{array}$ \\
\hline $\begin{array}{l}\text { Qualitative data } \\
\text { (patients/GPs/ }^{\text {paramedics* }} \text { ) }\end{array}$ & Interview & 3 months \\
\hline $\begin{array}{l}\text { Qualitative data } \\
\text { (paramedics) }\end{array}$ & Observation & $\begin{array}{l}\text { Throughout } \\
\text { recruitment }\end{array}$ \\
\hline \multicolumn{3}{|c|}{$\begin{array}{l}\text { *paramedic interviews will be carried out anytime throughout } \\
\text { recruitment. } \\
\text { EQ-5D-5L, Euroqol questionnaire; FRAX, Fracture Risk } \\
\text { Assessment Tool; GP, general practitioner. }\end{array}$} \\
\hline
\end{tabular}

4. The number, age, gender and relative rates of GP attendance, referral for investigation (DXA scan) or treatment (NICE or NOGG) between the intervention and control groups.

\section{Data management}

Data is stored and backed up on NHS passwordprotected computers within secured offices. Patient confidentiality will be protected by anonymising the data and allocating study numbers. Further, specific details of data management and patient confidentiality can be obtained from the corresponding author.

\section{Statistical analysis}

For this feasibility study percentages will be calculated with 95\% confidence limits using the exact binomial method. OR of being treated and/or investigated for falls and osteoporosis will be calculated with $95 \%$ CIs to provide a range of likely effect sizes to inform the full trial. Demographic data will be tabulated for those recruited to the study, for those who meet the exclusion criteria and for those unwilling to take part. Analysis will be carried out on data collected prior to patient discontinuation.

\section{Health economics methods and analysis}

This study will pilot the methodology to carry out a costeffectiveness analysis in the future trial. The future economic evaluation will include a cost-consequences study at 3 months, comparing costs and outcomes for patients in the short-term, and, if possible, a cost-effectiveness model to extrapolate costs and health benefits to the longer term, incorporating the possible effect of the intervention in fractures averted.

In this study we will assess the feasibility of collecting the resource-use data by reporting on the percentage of completed sets of data returned from patients and from research staff in case report forms. We will value resources required to deliver the intervention (training paramedics, administering the FRAX score and liaising with GPs) using national estimates for health and social care $^{20}$ or local sources when appropriate.

Each health status score produced by the EQ-5D-5L questionnaire will be allocated a preference-based quality-of-life weight for the UK population at baseline and 3 months. This allows quality-adjusted life years (QALYs) to be derived. A QALY weighs survival by the quality of life of the patient within that period of time. We will compute a 3-month incremental QALY using the area under the curve method and then adjust the QALY gain against baseline utility using linear regression with robust SEs. ${ }^{21}$

\section{Qualitative methods}

The qualitative study aims to assess the acceptability and feasibility of the intervention with patients, GPs and paramedics. Data collection will involve semistructured interviews with patients (up to 40), GPs (up to 20) and 
paramedics (up to 20) as well as observation of paramedics when attending people who have fallen (8). With informed consent, interviews will be digitally recorded and transcribed verbatim. The interviewer/observer will be an experienced qualitative health researcher.

Semistructured interviews with patients will elicit their views about the study design, including being asked the FRAX questions by ambulance crew, the recruitment process, the process of randomisation and its effect on differential treatment, and the acceptability of completing questionnaires and accessing their medical records 3 months post follow-up. Interview participants will be selected purposively to include a range of patients from intervention and control groups, genders, ages. Family carers will be included for their views where appropriate.

GPs who have received the intervention letters, with details of a patient's fracture risk and advice about treatment, will be interviewed to elicit their views on the trial design and the intervention. GPs will be asked about their current practice in fracture prevention, their opinions about the information received from the study team, whether and why they chose to follow recommendations and suggestions for improvement to the intervention and study design. GPs will be purposively selected based on whether they had patients in the intervention arm of the trial.

Selected (and consenting) participating paramedics will be observed in the course of their daily routine to help assess the feasibility of the trial processes and FRAX administration. Triangulating both these methods and data sets will more accurately assess the acceptability for paramedics to use FRAX in prehospital care, with people who have fallen. ${ }^{22}$ In interviews, paramedics will be asked about their experience of using FRAX with their patients who have fallen; their reflections on the recruitment process; the study training and reminders provided; the ease, or otherwise, of assessing mental capacity, particularly with regard to research and any other views on patients who fall.

When observing paramedics, notes will be made during and after the observation. ${ }^{23}$ Observations will take place throughout the recruitment period, examining the ease of asking the FRAX questions, the quality of information recorded, whether any barriers exist to carrying out the intervention and recruitment, whether any conversations take place about fracturing and falling prevention, whether any self-care advice is given, if any emotional support is given to patients, and how interagency relationships with other organisations work. Paramedics will be purposively sampled based on gender and level of experience.

The 3 sets of data (patients, GPs and paramedics) will be analysed separately with the aid of NVivo (V.10). Data will be inductively coded thematically, producing thematic frameworks. ${ }^{24} 20 \%$ of the transcripts and coding framework will be double-coded by another experienced qualitative researcher to ensure dependability and credibility of findings. ${ }^{25}$ The thematic coding frame will be explored and refined ${ }^{24}$ and findings will be interpreted in the light of existing theory and literature. The qualitative study will provide information about the acceptability of study processes and the intervention.

\section{DISCUSSION}

Although the majority of patients who experience a fragility fracture have done so consequent to a fall, and there are assessment algorithms that allow us to predict which patients are most likely to benefit from specific antiosteoporotic treatment, there is no mechanism within the NHS that allows for fracture risk assessment at the time of a fall. Fractures can be painful, disabling and are expensive to our health system.

This study is designed to collect data to help determine whether it may be feasible to link falls with fracture risk at the time a patient asks for help. It addresses the practicalities of teaching, collecting and delivering the FRAX information to a patient's GP and the acceptability of the intervention to the patients, paramedics and GPs. The study will collect data on the number of patients who fall and are at increased risk of fracture, and the proportion of those patients who are not already on treatment.

These data will allow calculation of the size of a subsequent RCT to determine whether paramedics, by assessing fracture risk in those who fall, can positively influence the number of fragility fractures that occur over a 5-year period.

\section{Dissemination}

The final data set will be held by the principal investigator but will be available on request (with patient identifiers removed to ensure participant confidentiality). The project outcomes will be relayed to all participants, the sponsor and funding body. The outcomes will be submitted (with the sponsor and funders approval) to peerreviewed journals and for presentation at relevant scientific local and national meetings. A publication plan and authorship criteria have been preagreed by the research team.

\section{Study management}

The principle investigator will have overall responsibility for project delivery.

A Project Steering Committee (PSC) will be independently chaired, will meet twice annually and be constituted by a majority of independent members who will be representatives from stakeholder groups (patients, ambulance service, academic research, general practice and secondary care). A Patient Advisory Group (PAG) will be established and meet 6 monthly, prior to the PSC. The terms of reference for the PSC and PAG are available from the corresponding author.

A Project Team will meet monthly but also maintains regular contact outside these times. 


\section{Adverse events}

Adverse events will be recorded and the study monitored and audited in accordance with Trust and Research Ethics Committee Guidance.

\section{Ethics approval and trial registration}

Amendments/modifications to the protocol have and will only be carried out after agreement from all investigators, the sponsor, funder and with ethical approval.

Although the study was registered with UKCRN on 5 March 2013, there was a delay in registering for an ISRCTN number, granted 13 March 2014. The trial was originally registered as qualitative. When it became clear ISRCTN registration would be appropriate, staffing issues at National Institute for Health Research (NIHR) led to a delay in receiving the correct advice.

\section{Protocol amendments}

The protocol described here is V.7. From March 2013 a number of amendments have been approved by the ethics committee: April 2013 the name of the Ambulance Trust was changed on the study documentation, ineligibility criteria were added for patients' resident outside the catchment area and the protocol was revised into Consort format. In October 2013 the option of face-to face recruitment was added and we were also granted permission to train clinical desk paramedics at a city call centre to request verbal consent from patients who called with a fall (this variation to the protocol was not actioned). In December 2013, references to 'ambulance paramedic' were changed to 'paramedic'.

\section{Current trial status}

Recruitment is planned to complete on 1 July 2014.

\author{
Author affiliations \\ ${ }^{1}$ University Hospitals NHS Foundation Trust, Bristol, UK \\ ${ }^{2}$ Centre for Academic Primary Care, School of Social and Community \\ Medicine, University of Bristol, Bristol, UK \\ ${ }^{3}$ Faculty of Health and Applied Sciences, University of the West of England, \\ Bristol, UK \\ ${ }^{4}$ School of Social and Community Medicine, University of Bristol, Bristol, UK \\ ${ }^{5}$ Norwich Medical School University of East Anglia, Norwich, UK \\ ${ }^{6}$ South Western Ambulance Service NHS Foundation Trust, Exeter, UK \\ ${ }^{7}$ School of Clinical Sciences, University of Bristol, Learning and Research \\ Building, Southmead Hospital, Bristol, UK
}

Acknowledgements All the authors acknowledge and are grateful for the support of South West Ambulance Service NHS Foundation Trust (and the former Great Western Ambulance Service), the Research Design Service and University Hospitals Bristol NHS Foundation Trust for support in designing and carrying out this study. The authors, and the study design, have benefitted from the help and advice of their patient partners (the Oak Leaves) to whom they are indebted. They acknowledge also the support of the National Institute for Health Research, through the Comprehensive Clinical Research Network. Finally, they would like to acknowledge the help of all those patients who participate in this research.

Contributors SC, RB, RG-H, RG, EM, CS, JB, LS and JA-F designed the study. BS collected the data, contributed to the qualitative work design, made ethical committee amendments and facilitated the research. MR facilitated links between the research team and the ambulance trust. SC, RG-H and BS drafted the article. All authors read and made critical revisions to the text before giving approval for the final version to be published.

Funding This paper presents independent research funded by the National Institute for Health Research (NIHR) under its Research for Patient Benefit (RfPB) Programme (Grant Reference Number PB-PG-0711-25070). The views expressed are those of the author(s) and not necessarily those of the NHS, the NIHR or the Department of Health.

Competing interests None.

Patient consent Obtained.

Ethics approval NRES Committee South Central Oxford C in October 2012. Research Ethics Committee reference 12/SC/0604.

Provenance and peer review Not commissioned; internally peer reviewed.

Data sharing statement Full, but anonymised, data will be available, on request from the corresponding author.

Open Access This is an Open Access article distributed in accordance with the Creative Commons Attribution Non Commercial (CC BY-NC 4.0) license, which permits others to distribute, remix, adapt, build upon this work noncommercially, and license their derivative works on different terms, provided the original work is properly cited and the use is non-commercial. See: http:// creativecommons.org/licenses/by-nc/4.0/

\section{REFERENCES}

1. Treml J. On behalf of the National Audit of Falls and Bone Health: Falling Standards, Broken Promises-report of the national audit of falls and bone health in older people 2010 (available as Falls and Bone Health National Report Executive Summary May 2011: Falls-Bone-Health-Report-2011-Full.pdf at http://www.rcplondon.ac. uk/resources/national-audit-falls-and-bone-health-older-people

2. Johnell O, Kanis J. Epidemiology of osteoporotic fractures Osteoporos Int 2005;16(Supp 2):S3-7.

3. Yoshida S. A global report on falls prevention: epidemiology of falls. World Health Organisation, 2007. http://www.who.int/ageing/ publications/Falls_prevention7March.pdf (accessed Aug 2013).

4. Stalenhoef PA, Crebolder HFJM, Knottnerus JA, et al. Incidence, risk factors and consequences of falls among elderly subjects living in the community: a criteria-based analysis. Eur J Public Health 1997;7:328-34

5. Copeland C, Wallace R, Anderson M, et al. Can information technology (IT) solve the issue of fallers presenting to Accident and Emergency ( \& E)? British Geriatric Society Papers submitted to annual scientific meeting 2009 (November): doi: 10.1093/ageing/afp229

6. Geusens P, Autier P, Boonen S, et al. The relationship among history of falls, osteoporosis, and fractures in postmenopausal women arch. Phys Med Rehab 2002;83:903-6.

7. Borgström $\mathrm{F}$, Johnell $\mathrm{O}$, Kanis $\mathrm{JA}$, et al. At what hip fracture risk is it cost-effective to treat? International intervention thresholds for the treatment of osteoporosis. Osteoporos Int 2006;17:1459-71.

8. Kanis JA, Brazier JE, Stevenson M, et al. Treatment of established osteoporosis: a systematic review and cost utility analysis. Health Technol Assess 2002;6:1-46.

9. Masud T, Binkley N, Boonen S, et al.; FRAX(囚) Position Development Conference Members. Official positions for FRAX(®) clinical regarding falls and frailty: can falls and frailty be used in FRAX( $($ ) ? From Joint Official Positions Development Conference of the International Society for Clinical Densitometry and International Osteoporosis Foundation on FRAX(囚). J Clin Densitom 2011;14:194-204.

10. Snooks H, Halter M, Close JT, et al. Emergency care of older people who fall: a missed opportunity. Qual Saf Health Care 2006;15:390-2.

11. Chapuy MC, Arlot ME, Duboeuf F, et al. Vitamin D3 and calcium to prevent hip fractures in the elderly women. $N$ Engl $J$ Med 1992;327:1637-42.

12. National Osteoporosis Guideline Group. http://www.shef.ac.uk/ NOGG/index.html (accessed Aug 2013).

13. NICE Technology Appraisal Guideline 160. http://guidance.nice.org. uk/TA160 (accessed Aug 2013).

14. NICE Technology Appraisal Guideline 161. http://guidance.nice.org. uk/TA161 (accessed Aug 2013).

15. NICE Technology Appraisal Guideline 204. http://guidance.nice.org. uk/TA204 (accessed Aug 2013).

16. NICE Clinical Guideline 161. http://guidance.nice.org.uk/CG161 (accessed Aug 2013).

17. FRAX® Home Page. http://www.shef.ac.uk/FRAX/ (accessed Aug 2013) 
18. Euroqol EQ-5D Questionnaire-access: http://www.euroqol.org/

19. Newton JL, Jones DE, Wilton $\mathrm{K}$, et al. Calcaneal bone mineral density in older patients who have fallen. QJM 2006;99:231-6.

20. Curtis L. Unit Costs of Health and Social Care PSSRU. 2010. http:// www.pssru.ac.uk

21. Manca A, Hawkins N, Sculpher MJ. Estimating mean QALYs in trial-based cost-effectiveness analysis: the importance of controlling for baseline utility. Health Econ 2005;14:487-96.
22. Flick U. An introduction to qualitative research. 3rd edn. London: SAGE, 2006.

23. Mays N, Pope C. Qualitative research: observational methods in health care settings. BJM 1995;311:182-4.

24. Attride-Stirling J. Thematic networks: an analytic tool for qualitative research. Qual Res 2001;1:385-405.

25. Patton MQ. Enhancing the quality and credibility of qualitative analysis. Health Serv Res 1999;34:1189-208. 